\title{
Maintaining an Adult Hematology/Oncology Service at a Tertiary Care Center during the SARS-CoV-2 Pandemic: An Eight-Week-Experience with a Newly Implemented Procedural Plan
}

\author{
Philipp G. Hemmati ${ }^{a}$ Dorothea Fischer ${ }^{b, f}$ Frank Breywisch ${ }^{a}$ Sabine Wohlfarth ${ }^{a}$ \\ Matthias Kramer ${ }^{a}$ Matthias Paland ${ }^{c}$ Lukas Tometten $^{d}$ Harun Badakhshi ${ }^{e}{ }^{f}$ \\ Gerald Ripbergerg, $^{\mathrm{h}}$ Georg Maschmeyer ${ }^{\mathrm{a}} \mathrm{f}$ \\ a Department of Hematology, Oncology und Palliative Care, Klinikum Ernst von Bergmann, Potsdam, Germany; \\ ${ }^{b}$ Department of Gynecology and Obstetrics, Klinikum Ernst von Bergmann, Potsdam, Germany; ${ }^{\mathrm{C}}$ Department \\ of Pneumonology, Klinikum Ernst von Bergmann, Potsdam, Germany; ${ }^{\mathrm{d}}$ Department of Gastroenterology and

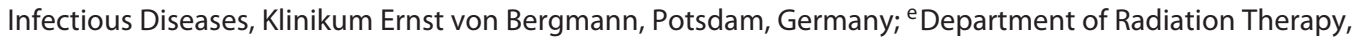 \\ Klinikum Ernst von Bergmann, Potsdam, Germany; ${ }^{\mathrm{f} C o m p r e h e n s i v e ~ C a n c e r ~ C e n t e r, ~ K l i n i k u m ~ E r n s t ~ v o n ~ B e r g m a n n, ~}$ \\ Potsdam, Germany; ${ }^{9}$ Department of Emergency and Intensive Care Medicine, Klinikum Ernst von Bergmann,

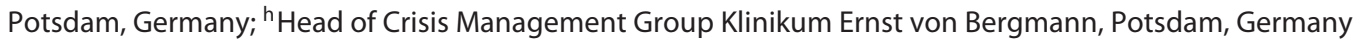

\section{Keywords}

SARS-CoV-2 COVID-19 P Pandemic $\cdot$ Procedural plan

\begin{abstract}
Treatment of cancer patients has become challenging when large parts of hospital services need to be shut down as a consequence of a local COVID-19 outbreak that requires rapid containment measures, in conjunction with the shifting of priorities to vital services. Reports providing conceptual frameworks and first experiences on how to maintain a clinical hematology/oncology service during the severe acute respiratory syndrome coronavirus 2 (SARS-CoV-2) pandemic are scarce. Here, we report our first 8 weeks of experience after implementing a procedural plan at a hematology/oncology unit with its associated cancer center at a large academic teaching hospital in Germany. By strictly separating team workflows and implementing vigorous testing for SARS-CoV-2 infections for all patients and staff members irrespective of clinical symptoms, we were successful in maintaining a comprehensive hematology/oncology service to allow for the continuation of treatment for our patients. Notably, this was achieved without introducing or further trans-
\end{abstract}

mitting SARS-CoV-2 infections within the unit and the entire center. Although challenging, our approach appears safe and feasible and may help others to set up or optimize their procedures for cancer treatment or for other exceedingly vulnerable patient cohorts.

(c) 2021 S. Karger AG, Basel

\section{Introduction}

After its first occurrence in Wuhan (Hubei, China) in December 2019, infections with SARS-CoV-2 rapidly evolved into a pandemic. As of July 5, 2020, a total of $11,279,465$ SARS-CoV-2 cases had been confirmed worldwide and 531,056 deaths were reported from 188 countries or regions [1]. The SARS-CoV-2-associated disease, termed corona virus disease 2019 (COVID-19), not only involves emergency and intensive care medicine. COVID-19 is a complex inflammatory disorder which, in addition to affecting the respiratory tract, is associated with a wide range of conditions including cardiovascular disease, autoimmune phenomena, coagulopathies, neurological defects, and others $[2,3]$. This in turn demands 
the specific expertise of medical specialties across all age groups.

Patients with active cancer represent an exceedingly vulnerable cohort as they are usually more advanced in age and carry relevant comorbidities. Patients with an underlying malignancy were reported to be more prone to infections, both in terms of frequency and severity [4]. Specifically, the risk for developing severe COVID-19 appears to be higher in cancer patients [5]. Whether the increased mortality is cancer-specific or is rather related to the general risk factors more frequently experienced by cancer patients is not clear yet $[6,7]$. Notably, a recent report indicated that the prognosis of cancer patients with COVID-19 is not inferior to that of people without cancer [8].

Recently, it was proposed that specialized cancer centers should strongly consider developing strategic plans for the management of their patients $[9,10]$. First results of specific measures covering a 4 -week period were reported by the National University Cancer Institute in Singapore and a Cancer Center in Changsha, China $[11,12]$. Recently, the implications of a COVID-19 outbreak in terms of clinical care at the University of Hamburg Cancer Center, Germany, were reported in detail [13]. However, numerous unanswered questions remain. What can be done to prevent SARS-CoV-2 infections in a cancer patient? Should all cancer patients and their medical caregivers be screened in the absence of COVID-19 symptoms, and, if so, at what frequency? Should a treatment be given or postponed, and, if so, for whom and for how long?

Here, we report the first 8 weeks of experience after implementing a specific set of procedures to maintain a hematology/oncology service at a German academic medical center during the SARS-CoV-2 pandemic.

\section{Background and Clinical Services}

Our center, the Klinikum Ernst von Bergmann (KEvB), serves as a tertiary care regional hospital. In 2018, the total number of hospital beds was 1,126 , and we treated 40,915 inpatients, 3,261 semi-inpatients, and 83,278 outpatients. KEvB provides general services and is a reference center for a wide range of specialized medical procedures. In addition to the main campus located in the city of Potsdam, 2 smaller, associated sites are operated in surrounding counties. There is a close collaboration with community hospitals in the area. Furthermore, our center is affiliated to the Charité University Medical Center in Berlin, as a teaching hospital. Patients admitted are primarily from the Berlin-Potsdam metropolitan area, with approximately 6 million inhabitants. However, patients from more remote rural areas are also referred to our institution.
Medical treatment in the department of hematology, oncology and palliative care (a total capacity of 66 beds for inpatients) covers the full spectrum of malignant and nonmalignant diseases, except for allogeneic hematopoietic stem cell transplantation and cellular immunotherapy. In 2018 , we treated 1,942 inpatients and 2,198 semiinpatients. The department also provides a day hospital and an ambulatory care facility which hosts 24 treatment stations. In 2018, a total of 9,818 patients were registered, including those receiving follow-up care or seeking medical advice.

\section{Implementation of Specific Procedural Plan}

After an inhouse SARS-CoV-2 outbreak with a total of 379 infected individuals (165 patients and 214 medical caregivers), local authorities mandated a lockdown of all admissions effective from 1 April 2020. Urgent medical procedures allowing no delays were maintained in the departments of obstetrics, neonatology and hematology, oncology, and palliative care. This required the implementation of a strategic plan to minimize the risk of a further spread of SARS-CoV-2 infections from patient to patient as well as from medical caregivers to patients and vice versa. We therefore developed a procedural plan as depicted in Figure 1. Central to this approach is a strictly tripartite team workflow and vigorous testing for SARS$\mathrm{CoV}-2$ by oropharyngeal (OP) smears, respectively. The latter was performed using a rapid nucleic acid-based detection assay (NADA) aiming at a turnaround time ranging from $4 \mathrm{~h}$ to a maximum of $24 \mathrm{~h}$. Specifically, 4 different PCR-based test platforms were used: VIASURE SARS-CoV-2 S gene real-time PCR detection kit (CerTest Biotec, Zaragoza, Spain), BD SARS CoV-2 test kit for BD MAX (Becton Dickinson, Heidelberg, Germany), Xpert Xpress SARS-CoV-2-kit (Cepheid GmbH, Krefeld, Germany), and VitaPCR SARS-CoV2 test assay (Credo Diagnostics Biomedical, Singapore). Tests were performed irrespective of clinical symptoms of COVID-19, e.g., cough, coryza, sore throat, and shortness of breath, or systemic symptoms such as fever, malaise, or myalgia in all patients scheduled for admission. To this end, a testing facility was established. During March and April, this facility was located on a separate floor on the ground level of the main building. Patients were able to enter/exit at the street level via an extra entrance/exit. By the end of April, the testing facility had been moved to a separate building located on the edge of the hospital campus. The swabs were taken by members of the hospital staff. The vast majority were recruited from the nursery teams. All staff members were trained in taking swabs by the Department of Hygiene and Microbiology. Taking OP swabs was in accordance with the local guidelines proposed by the Department of 


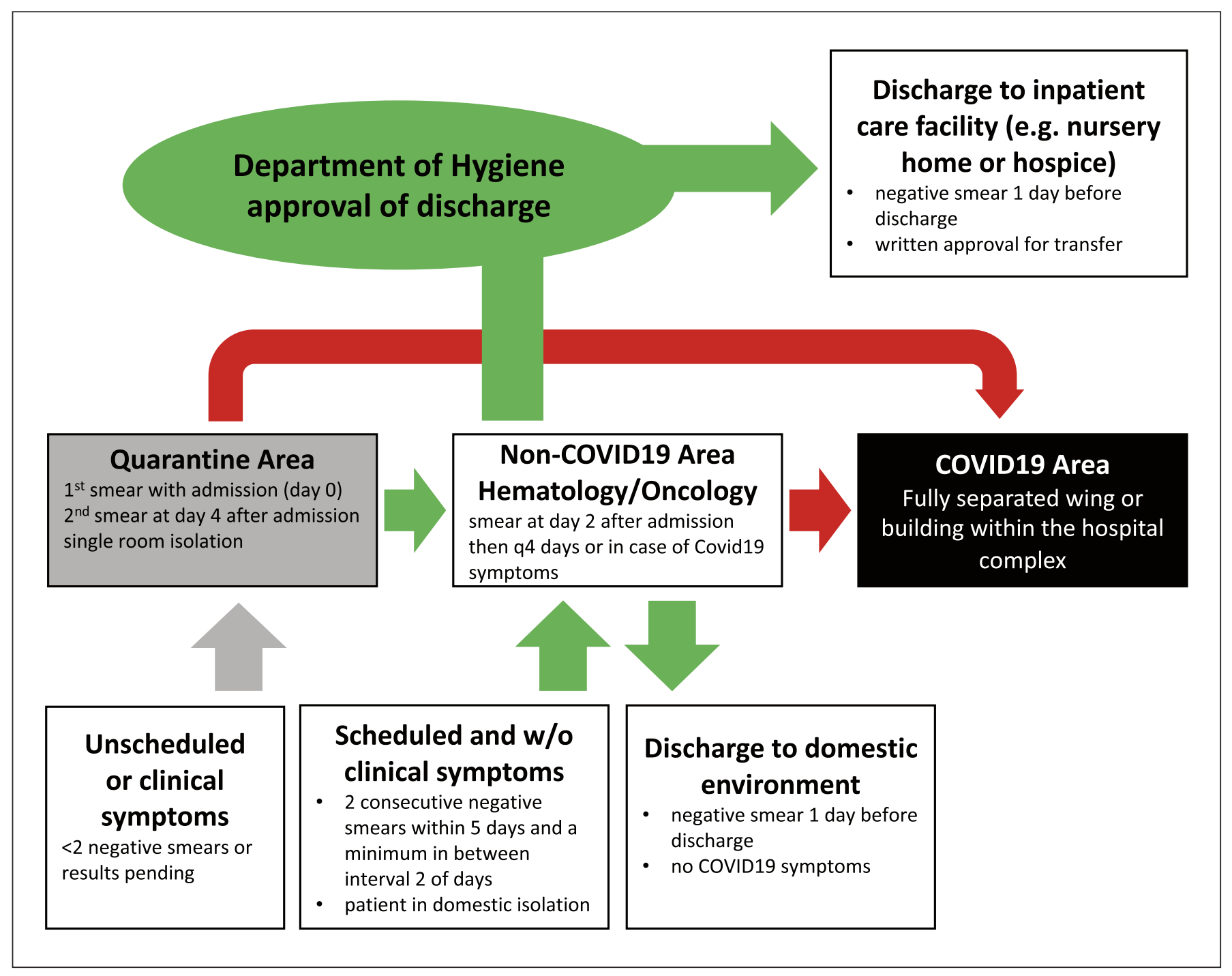

Fig. 1. Procedural plan for admission/discharge of inpatients. Admission/discharge and transfer of patients between the different areas is indicated by arrows. Green arrow, negative SARS-CoV-2 smear; grey arrow, results of SARS-CoV-2 smear pending; red arrow, positive SARS-CoV-2 smear.

Hygiene and Microbiology and was approved by the local health authorities. Taking OP swabs rather than nasopharyngeal (NP) swabs was also supported by an external panel of experts who served as medical consultants.

Notably, patients were advised to stay in domestic isolation after the first smear. A minimum of 2 consecutive negative SARS-CoV-2 tests within 5 days (a minimum interval between $48 \mathrm{~h}$ ) was required for admission to the "Non-COVID Area", e.g., 1 of the 3 hematology/oncology units. In all inpatients, stringent testing for SARS-CoV-2 was continued at a fixed schedule, i.e., every 4 days for patients without clinical signs of COVID-19 or immediately at the onset of suspicious symptoms or radiographs/computed tomography (CT) scans during hospital stay. Testing at the same frequency was performed in all medical caregivers, i.e., physicians, nurses, and paramedic staff delivering direct patient care as well as physiotherapist and psychologists. The same was true for all personnel not involved in patient-centered care (e.g., food service, cleaning, and housekeeping) who entered the patients' rooms. Further measures included strict single room accommodation and the use of a protective mask whenever close $(<1.5 \mathrm{~m})$ or in direct contact with the patient. In patients lacking 2 negative consecutive smears, due to an unscheduled admission or pending test results, and those with suspicious clinical symptoms, direct admission to the "NonCOVID Area" was prohibited. Instead, depending on the urgency and the severity of symptoms, patients were admitted to a "Quarantine Area." By definition, all patients in this area were classified as potentially contagious, so full protective measures (wearing a gown or overall, an FFP2/3 mask, gloves, and full-face shield with eye cover) were applied at all times. Single room accommodation was mandatory. Transit to the "Non-COVID Area" was allowed 
only after 2 negative SARS-CoV-2 tests (performed at admission and at day +4 ). In addition to the "Non-COVID Area" and the "Quarantine Area," a "COVID Area" was established. This was located in a separate building or wing of the hospital and also included 2 fully equipped and operational intensive care units. In the case of a positive SARS-CoV-2 test, patients were transferred to this area. Discharge from the hospital also required defined precautions. Specifically, transfer of patients to nursery homes or other shared facilities like hospices required approval by the Department of Hygiene and the local health authorities, respectively.

The hematology/oncology day clinic and outpatient care facility were moved to a separate floor of the hospital equipped with an extra registration counter, triage area, venipuncture service, consultation rooms, and 24 treatment stations. For all outpatients, a single negative SARSCoV-2 smear (maximum $72 \mathrm{~h}$ before admission was scheduled) was required for admission. Physicians and nurses were not strictly segregated between the inpatient and the day hospital/outpatient facility teams. However, all other cross-hospital staff transfer was fully suspended. The Department of Radiotherapy and its associated ambulatory facility is operated by a separate team which comprises radiation oncologists, radiation therapists, physicists, nurses, and administrative staff.

Between March 30 and May 25, 2020, a total of 1,557 patients were treated at the hematology/oncology day hospital and outpatient care facility. Services were restricted to patients under active treatment with intravenous chemo- and/or immunotherapies. For other patients, i.e., those receiving oral therapeutics or follow-up visits after completion of therapy, telephone consultations were established. Patients referred from other centers or primary care physicians and those seeking secondary medical advice were, depending on urgency, either admitted to the inpatient service, transferred to another specialized center, or deferred.

Further general procedures at our center included mandatory wearing of a surgical mask (covering the mouth and nose) at all times by all hospital staff members. Patients were also advised to wear a surgical mask when leaving their rooms. From 14 April onwards, visitors were generally not permitted. Exceptions were made for the Departments of Palliative Care, Obstetrics and Neonatology (1 visitor/day for a maximum interval of $1 \mathrm{~h}$ was allowed). Likewise, individual exceptions were granted for visitors to critically ill patients. All visitors had to fill out a self-assessment form. Clinical rounds were reduced to a minimum of once daily, meetings and conferences were restricted to 1 specialist per field, and journal clubs as well as trainee programs for medical students and residents/ fellows were suspended or held as tele-conferences or video broadcasts only.

Hematology/Oncology Service during SARS-CoV-2 Pandemic
During an 8-week interval, a total of 235 patients were treated as inpatients. Of note, all dose-intensive therapies, e.g., those for acute leukemias, and high-dose chemotherapy followed by autologous stem cell transplantation (HDT/autoSCT), were performed as usual. In keeping with this strategy, over time, we did not note a substantial difference in the types of therapy given or withheld. For outpatients, the telephone visits were increased so as to, at least in part, compensate for personal visits. Furthermore, there was a decline in the number of patients scheduled for laboratory tests or imaging studies from April to June. At the first round of SARS-CoV-2 testing performed on 28 March 2020, 2 patients were tested positive. Patient 1 (a 43-year-old female with acute myeloid leukemia receiving a second course of high-dose cytarabine for consolidation and entering the neutropenic phase tested positive for SARS-CoV-2) was transferred to the "COVID Area" of the hospital. She did not develop any symptoms of COVID-19 and was discharged at day 17. Patient 2 (an 83-year-old male with diffuse large B cell lymphoma on the second course of immunochemotherapy and with severe chronic pulmonary disease, was in the neutropenic phase when he tested positive for SARS-CoV-2) also had to be transferred to the "COVID-Area." He developed a severe form of COVID-19, and, after rapidly progressive worsening of his pulmonary symptoms, rejected intubation and mechanical ventilation. He died from pulmonary failure at day 8 after the positive SARS-CoV-2 test. Two members of the medical staff also tested positive during the first test round. Both only had minor symptoms of an infection and were sent into domestic quarantine immediately. As only mild signs of COVID-19 developed thereafter, no specific treatment was necessary. At all consecutive test rounds (a total number of tests $>22,000$ ), there were no further positive tests for SARSCoV-2 in patients or medical and paramedical staff. Two outpatients tested positive for SARS-CoV-2 and were sent into domestic quarantine. Neither of them was under active treatment and they were scheduled for regular follow-up visits. No member of staff tested positive between 30 March and 3 July, 2020.

\section{Discussion}

Cancer patients require special precautions to prevent severe infections. Furthermore, deviating from established treatments protocols or postponing specific procedures may have deleterious effects on outcome and jeopardize their physical and mental health. In this regard, the SARS-CoV-2 pandemic and the associated COVID-19 represent an unprecedented medical challenge, prompting many specialized centers to initiate specific plans and procedures to tackle this problem $[9,10,12]$. A recently 
published report from the University Cancer Center in Hamburg (Germany) has enabled a detailed insight into the procedures at a single institution; it can serve as a template for other institutions to develop similar strategies and interconnect and scientifically analyze the results [13]. Based on the available data, medical societies have published recommendations to guide clinicians in their daily practice [14]. However, so far, reports on the success of these strategies are scarce.

The primary goal of our approach was to maintain a hematology/oncology service under the condition of a local cluster of COVID-19 cases during the SARS-CoV-2 pandemic in Germany. This prompted the temporary and almost complete lockdown of services at our center. To this end, and to minimize the potential to introduce and transmit SARS-CoV-2 infections within our unit, we developed, implemented, and modified (step-by-step), a procedural plan which was essentially based on the strict separation of medical workflows between a "COVID Area," a "Non-COVID Area," and a "Quarantine Area." In this regard, our strategy resembles previously reported experiences from centers in Singapore and China $[11,12]$. This strategy has prevented the introduction and transmission of SARS-CoV-2 infections in our units. This is remarkable in the context of the SARS-CoV-2 outbreak that affected 379 individuals (165 patients and 214 staff members) noted at the entire medical center beginning on 24 March 2020. Of the patients treated in the COVID unit of our center, $31 \%(n=33)$ had a previous history of a malignancy (mainly a solid tumor). In $64 \%$ of these cases, the tumor was active, and $15 \%$ under a specific treatment at the time tested positive for SARS-CoV-2. The clinical characteristics and outcomes of these patients were fed into the LEOSS registry. A detailed analysis (as part of a larger cohort) was published recently by Rüthrich et al. [15].

Regarding the testing strategy that included asymptomatic individuals and frequency (i.e., every 4 days), our approach differed from the recommendations by both regional and national authorities which, at that point, did not generally advocate testing of asymptomatic individuals who had not had contact with an infected patient or who were returning from so-called "risk areas" (areas with a high number of infected individuals). However, this policy has been revised recently, possibly as a result of broader test availability and further reductions in turnaround time. Likewise, attempts to expand testing to asymptomatic individuals, in conjunction with an assay to detect neutralizing antibodies, may be relevant.

At our center, we chose a NADA-based serial testing approach at 4-day intervals for all patients and staff members, so as to identify asymptomatic virus carriers as early as possible and thereby further reduce the risk of transmitting SARS-CoV-2. In fact, this seems mandatory, as the total number of infected individuals including those with an asymptomatic infection is estimated as being 10 (range 5-20) times that of the confirmed cases [16]. This 4-day time frame was chosen because most published reports indicate a median incubation time of SARS-CoV-2 in the range of 4-6 days $[17,18]$. However, further work is needed to better define intervals. Also, the development of a herd immunity and/or the availability of an efficient vaccine will likely require further adaptions of the testing strategy. The general impression is that the frequent testing in our institution was perceived in a positive manner by both patients and health care professionals.

As of July 5, 2020, 197,388 individuals tested positive for SARS-CoV-2 in Germany and 9,022 deaths were reported. In the Berlin-Brandenburg area, 11,922 individuals infected with SARS-CoV-2 were identified, and a total of 387 deaths were noted in conjunction with a confirmed SARS-CoV-2 infection. This converts into 19.3 infected individuals/100.000 inhabitants, which is among the lowest in Germany. Although we cannot rule out other contributory factors, we speculate that the low positive rates in both patients and staff are in keeping with this low background incidence of SARS-VoV-2 infections in the general population. In this regard, our approach might not be adequate in regions with a different epidemiologic profile of SARS-CoV-2, e.g., the presence of substantially more infected individuals.

Lastly, it needs to be pointed out that the procedures implemented at our center during March and thereafter had tremendous implications regarding resources, i.e., financial (costs for repetitive testing of patients and staff and a reduced capacity for treatment of inpatients and outpatients), personnel (the provision of additional staff to allow for separate workflows and continuous adjustment of staff rosters), and others (internal and external communication, design and evaluation of new procedures and plans, and the purchase of devices and equipment). In sum, these constituted a substantial burden with respect to daily work and the provision of high-quality health care in general.

Taken together, in our center, the implementation of a procedural plan based on strict team segregation and stringent testing for SARS-CoV-2 in both patients and staff has been challenging but appears feasible and safe. This strategy was highly effective to reduce the occurrence of SARS-CoV-2 infections and associated COVID-19, and it allowed us to maintain a hematology/oncology service at our center. However, managing cancer patients during the SARS-CoV-2 pandemic remains a huge challenge. 


\section{Acknowledgement}

We would like to thank Tim Steckel for reviewing and commenting the manuscript. Furthermore, we would like to thank Evangelos Tsekos for providing detailed information on the SARSCoV-2 testing platforms used.

\section{Statement of Ethics}

All procedures followed were in accordance with the ethics standards of the responsible committee on human experimentation (institutional and national) and the Helsinki Declaration of 1975 , as revised in 2000 .

\section{Conflict of Interest Statement}

The authors declare no conflicts of interest.

\section{Funding Sources}

No funding was received.

\section{Author Contributions}

P.G.H., D.F., H.B., and G.M.: conceptional design. All authors contributed to the discussion of procedures, data analysis, and writing of the manuscript.

\section{References}

1 https://coronavirus.jhu.edu/map.html.

2 Guan WJ, Ni ZY, Hu Y, Liang WH, Ou CQ, He JX, et al.; China Medical Treatment Expert Group for Covid-19. Clinical Characteristics of Coronavirus Disease 2019 in China. N Engl J Med. 2020 Apr;382(18):1708-20.

3 Richardson S, Hirsch JS, Narasimhan M, Crawford JM, McGinn T, Davidson KW, et al.; the Northwell COVID-19 Research Consortium. Presenting Characteristics, Comorbidities, and Outcomes Among 5700 Patients Hospitalized With COVID-19 in the New York City Area. JAMA. 2020 May;323(20): 2052-9.

4 Kamboj M, Sepkowitz KA. Nosocomial infections in patients with cancer. Lancet Oncol. 2009 Jun; 10(6):589-97.

5 Liang W, Guan W, Chen R, Wang W, Li J, Xu $\mathrm{K}$, et al. Cancer patients in SARS-CoV-2 infection: a nationwide analysis in China. Lancet Oncol. 2020 Mar;21(3):335-7.

6 Kuderer NM, Choueiri TK, Shah DP, Shyr Y, Rubinstein SM, Rivera DR, et al.; COVID-19 and Cancer Consortium. Clinical impact of COVID-19 on patients with cancer (CCC19): a cohort study. Lancet. 2020 Jun;395(10241): 1907-18.
7 Lee LY, Cazier JB, Angelis V, Arnold R, Bisht V, Campton NA, et al.; UK Coronavirus Monitoring Project Team. COVID-19 mortality in patients with cancer on chemotherapy or other anticancer treatments: a prospective cohort study. Lancet. 2020 Jun; 395(10241):1919-26.

8 Wang H, Zhang L. Risk of COVID-19 for patients with cancer. Lancet Oncol. 2020 Apr; 21(4):e181.

9 Cortiula F, Pettke A, Bartoletti M, Puglisi F, Helleday T. Managing COVID-19 in the oncology clinic and avoiding the distraction effect. Ann Oncol. 2020 May;31(5):553-5.

10 Mussetti A, Maluquer C, Albasanz-Puig A, Gudiol C, Moreno-Gonzalez G, Corradini P, et al. Handling the COVID-19 pandemic in the oncological setting. Lancet Haematol. 2020 May;7(5):e365-6.

11 https://doi.org/10.1016/j.annonc.2020.03.306.

12 He Y, Lin Z, Tang D, Yang Y, Wang T, Yang M. Strategic plan for management of COVID-19 in paediatric haematology and oncology departments. Lancet Haematol. 2020 May;7(5):e359-62.

13 Weisel KC, Morgner-Miehlke A, Petersen C, Fiedler W, Block A, Schafhausen P, et al. Implications of SARS-CoV-2 Infection and COVID-19 Crisis on Clinical Cancer Care: Report of the University Cancer Center Hamburg. Oncol Res Treat. 2020;43(6):307-13.
14 von Lilienfeld-Toal M, Vehreschild JJ, Cornely O, Pagano L, Compagno F, Hirsch $\mathrm{HH}$; EHA Infectious Disease Scientific Working Group. Frequently asked questions regarding SARS-CoV-2 in cancer patients-recommendations for clinicians caring for patients with malignant diseases. Leukemia. 2020 Jun; 34(6):1487-94.

15 Rüthrich MM, Giessen-Jung C, Borgmann S, et al. COVID-19 in cancer patients: clinical characteristics and outcome-an analysis of the LEOSS registry. Ann Hematol. 2021 Feb; 100(2):383-93

16 Nishiura H, Kobayashi T, Yang Y et al. The Rate of Underascertainment of Novel Coronavirus (2019-nCoV) Infection: Estimation Using Japanese Passengers Data on Evacuation Flights. J Clin Med. 2020 Feb;9(2):419.

17 Linton NM, Kobayashi T, Yang Y et al. Incubation Period and Other Epidemiological Characteristics of 2019 Novel Coronavirus Infections with Right Truncation: A Statistical Analysis of Publicly Available Case Data. J Clin Med. 2020 Feb;9(2):538.

18 Backer JA, Klinkenberg D, Wallinga J. Incubation period of 2019 novel coronavirus (2019-nCoV) infections among travellers from Wuhan, China, 20-28 January 2020. Euro Surveill. 2020 Feb;25(5):2000062. 